\title{
Optimization Safety Factors to Study an Ultrasonic Motor
}

\author{
Bouchaib Radi ${ }^{1, \mathrm{a}}$, Abderahman Makhloufi ${ }^{2}$, Abdelkhalak El Hami ${ }^{2}$ and Mohamed Sbaa ${ }^{1}$ \\ ${ }^{1}$ FST Settat, BP : 577, route de Casablanca, Settat, Morocco \\ ${ }^{2}$ LMR, INSA de Rouen, BP: 8, Avenue de l'Université, 76801 St Etienne de Rouvray, France
}

Received 8 December 2009, Accepted 15 March 2010

\begin{abstract}
In this paper, we present a new method based on Optimal Safety Factors (OSF) in the context of the Reliability-Based Design Optimization (RBDO) analysis of ultrasonic motors with traveling wave taking into account the contact between the different components (stator and rotor). We will underline also the different methods of the RBDO analysis and we highlight the advantage of our approach based on OSF. Numerical results are given to illustrate the proposed method.
\end{abstract}

Key words: Reliability based design optimization (RBDO), Random variable, Risk of failure, Contact, Optimal safety factors (OSF).

\section{Introduction}

Under normal conditions of operation, the ultrasonic motors are subjected to: (i) an axial static loading of pre-stressing producing axial and radial deformations in stator and rotor, (ii) a dynamic excitation of the stator, involving deformations of bending out of the plane, which is due to rigid displacement of the rotor's body and (iii) contact, static and dynamic friction efforts in the contact zone between the stator and the rotor.

The aim of this study is to propose a numerical modeling by the FE method of the mechanical behavior of an ultrasonic motor SHINSEI USR 60 pennies dynamic loading taking into account the contact without friction by using the reliability analysis. Generally, the life of these engines is limited by important abrasion of different components. So, the notion of random variables and the risk of failure must be integrated in the mechanical analysis to ensure the good working of the system.

Contact problems are treated using an augmented Lagrangian approach to identify the candidate contact surface and contact stresses [1]. The stochastic role of each design parameter in the default risk is highlighted. For this purpose, we propose a direct mechanic-reliability between the augmented Lagrangian method to solve the contact (development based on FE method) and the probabilistic method FORM [2,3].

In this paper, we present a new approach based on optimal safety factors (OSF) in the context of the RBDO analysis of the ultrasonic motor with traveling wave taking into account the contact between the different components (stator and rotor). Generally, the objective of the RBDO model is to help design of structures which should be both economic and reliable where the solution reduces the structural weight in uncritical regions. It does not only provide an improved de- sign but also a higher level of confidence in the design \cite $\{$ elhami1\}.

\section{Contact treatment}

Contact between solids is generally governed by the complementary condition in the contact zone $\mathrm{S}_{\mathrm{c}}$ :

$$
u_{n} \cdot r_{n}=0
$$

which can be written as follows:

$$
\left\{\begin{array}{lll}
u_{n}-\delta<0 & \Rightarrow & r_{n}=0 \\
u_{n}-\delta=0 & \Rightarrow & r_{n} \leq 0
\end{array}\right.
$$

where $\delta$ represents the gap between the contacting bodies, $\mathrm{u}_{\mathrm{n}}$ the normal component of the displacement field and $\mathrm{r}_{\mathrm{n}}$ the normal reaction [5].

From the Hamilton principle, the system energy and work of external forces can be written as follows:

$$
\begin{gathered}
\Pi(u)=\int_{V} \frac{1}{2} \varepsilon^{t} D \varepsilon d V-\int_{S_{1}} u^{t} R d S \\
-\int_{S_{2}} u^{t} R d S-\int_{S_{c}} u^{t} R_{c} d S-\int_{V} u^{t} P d V
\end{gathered}
$$

Enforcement of the zero-penetration condition on contacting boundaries yields: $T^{t} u-\delta \geq 0, \quad u \in S_{c}$ where $\varepsilon$ is the strain vector, $\mathrm{D}$ is the material matrix, $\rho$ is the mass density, $\mathrm{u}$ is the displacement vector, $\mathrm{P}$ is the external load vector, $\mathrm{R}$ is the reaction force vector on prescribed displacement boundary, $\mathrm{T}$ is the contact constraint matrix, $\mathrm{S}_{1}$ is the boundary with prescribed external forces, $\mathrm{S}_{2}$ is the boundary with prescribed displacements and $\mathrm{S}$ is the global surface.

\footnotetext{
${ }^{a}$ Corresponding author: bouchaib.radi@yahoo.fr
} 
The augmented Lagrangian approach relative to the contact problem is given by the weak form of the equilibrium state:

$$
\Pi^{*}(u, \delta u)=\Pi(u, \delta u)=\int_{S_{c}}\left(T^{t} \delta u-\delta\right)^{t} r_{n} d S
$$

where $\delta$ is the gap between two contacting bodies.

The nonlinear equation [5] cannot be solved directly because the unknown $u$ and $r_{n}$ are implicitly dependent, so $r_{n}$ is considered as known via an iterative process using the Newton-Raphson method. The reaction is written as function of a Lagrange multiplier $\lambda$ and a penalty coefficient $\epsilon$ as follows:

$$
r_{n}=\lambda+\varepsilon u_{n}
$$

\section{Reliability design optimization}

\subsection{Classical approach}

Traditionally, for the reliability-based optimization procedure we use two spaces: the physical space and the normalized space (see figure 1a) [6]. Since very many repeated searches are needed in the above two spaces, the computational time for such an optimization is a big problem. To overcome these difficulties, two points of view have been considered.

RBDO involves the evaluation of probabilistic constraints, which can be executed in two different ways: either using the Reliability Index Approach (RIA), or the Performance Measurement Approach (PMA) [7,8]. Recently, the enhanced hybrid mean value method is proposed to improve numerical stability and efficiency in the Most Probable Point (MPP) search [9]. The major difficulty lies in the evaluation of the probabilistic constraints, which is prohibitively expensive and even unpractical for many applications [10].

However, from the optimization view point, an efficient method called the Hybrid Method (HM) has been elaborated by [11] where the optimization process is carried out in a Hybrid Design Space (HDS) (exposed in section 3.2). This method has been shown to verify the optimality conditions relative to the classical RBDO method.

Therefore, the reliability-based optimization is performed by nesting the two following problems:

i) Optimization problem:

$$
\begin{array}{cc}
\min & f(x) \\
\text { subject } & g_{k}(x) \leq 0, \beta(x, u) \geq \beta_{t}
\end{array}
$$

where $\mathrm{f}(\mathrm{x})$ is the objective function, $g_{k}(x) \leq 0$ are the associated constraints, $\beta(x, u)$ is the reliability index of the structure, and $\beta_{t}$ is the target reliability.

ii) Reliability analysis: The reliability index $\beta(x, u)$ is determined by solving the minimization problem:

$$
\begin{gathered}
\beta=\min \quad \operatorname{dis}(u) \\
\text { subject } H(x, u) \leq 0
\end{gathered}
$$

where $\operatorname{dis}(u)=\sqrt{\sum_{j=1}^{m} u_{j}^{2}}$ is the distance in the normalized random space and $\mathrm{H}(\mathrm{x}, \mathrm{u})$ is the performance function (or limit state function) in the normalized space, defined such that $\mathrm{H}(\mathrm{x}, \mathrm{u})<0$ implies failure, see figure 1 . In the physical space, the image of $\mathrm{H}(\mathrm{x}, \mathrm{u})$ is the limit state function $\mathrm{G}(\mathrm{x}, \mathrm{y})$, see figure 1 . The solution of these nested problems leads to very large computational time, especially for large-scale structures.

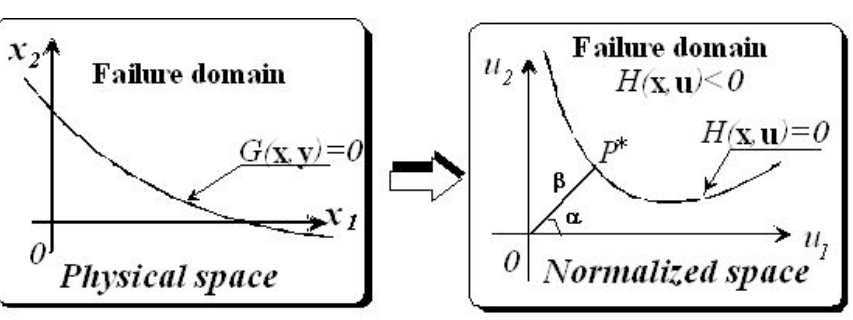

Fig. 1. Transformation between the physical space and normalized one.

\subsection{Hybrid approach}

In order to improve the numerical performance, the hybrid approach consists of minimizing a new form of the objective function $\mathrm{F}(\mathrm{x}, \mathrm{y})$ subject to a limit state and to deterministic as well as to reliability constraints:

$$
\begin{array}{cc}
\min & f(x) \cdot d_{\beta}(x, y) \\
& G(x, y) \leq 0 \\
\text { subject } & d_{\beta}(x, y) \leq \beta_{c} \\
& g_{k}(x) \leq 0
\end{array}
$$

$d_{\beta}(x, y)$ is the distance in the hybrid space between the optimum and the design point, $d_{\beta}(x, y)=d(u)$. The minimization of the function $\mathrm{F}(\mathrm{x}, \mathrm{y})$ is carried out in the Hybrid Design Space (HDS) of deterministic variables $x$ and random variables y.

An example of this HDS is given in figure 2, containing design and random variables, where the reliability levels $d_{\beta}$ can be represented by ellipses in the case of normal distribution, the objective function levels are given by solid curves and the limit state function is represented by dashed level lines except for $G(x, y)=0$. 
We can see two important points: the optimal solution $P_{x}^{*}$ and the reliability solution $P_{y}^{*}$ (i.e. the design point found on the curves $\mathrm{G}(\mathrm{x}, \mathrm{y})=0$ and $d_{\beta}=\beta_{t}$.

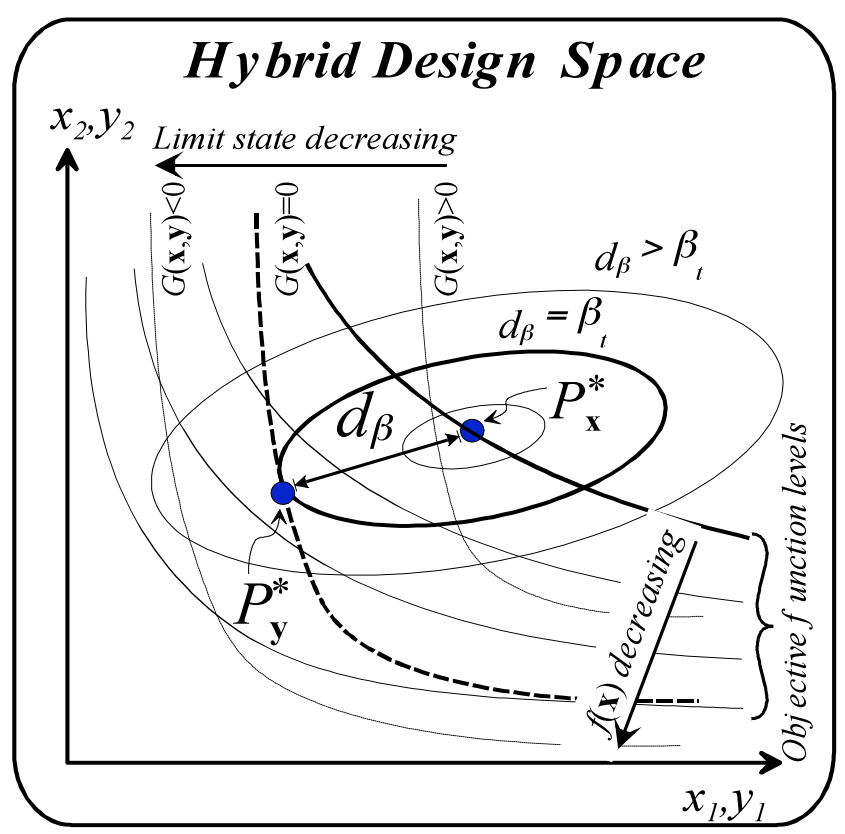

Fig. 2. Hybrid design space

In [11], it is demonstrated that the hybrid method (HM) reduced the computational time almost $80 \%$ relative to the classical RBDO approach. Using the HM, the optimization process is carried out in the Hybrid Design Space (HDS) where all numerical information in the case of the optimization process can be modeled. Furthermore, the classical method (CM) has weak convergence stability because it is carried out in two spaces (physical and normalized spaces). In fact, when using the HM, we have a complex optimization problem with many variables. This problem may converge into a local solution. To overcome these drawbacks (several local solutions), an optimum safety factor method has been proposed [12].

\subsection{Optimization based safety factors (OSF)}

In general, when considering the normal distribution law, the normalized variable $u_{i}$ is given by:

$$
u_{i}=\frac{y_{i}-m_{i}}{\sigma_{i}}, \quad i=1, \ldots, n
$$

where $y_{i}$ is the component of the vector $y$. The standard deviation $\sigma_{\mathrm{i}}$ can be related to the mean value $\mathrm{m}_{\mathrm{i}}$ by:

$$
\sigma_{i}=\gamma_{i} \cdot m_{i}, \quad \text { or } \quad \sigma_{i}=\gamma_{i} \cdot x_{i}, \quad i=1, \ldots, n
$$

We introduce the safety factors $S_{f_{i}}$ corresponding to the design variables $\mathrm{x}_{\mathrm{i}}$. The design point can be expressed by:

$$
y_{i}=S_{f_{i}} \cdot x_{i}, \quad i=1, \ldots, n
$$

For an assumed failure scenario $\mathrm{G}(\mathrm{y})<0$, the equation of the optimum safety factor for a single limit state case can be written in the following form $[13,14]$ :

$$
S_{f_{i}}=1 \pm \gamma_{i} \cdot \beta_{t} \sqrt{\frac{\left\|\frac{\partial G}{\partial y_{i}}\right\|}{\sum_{i=1}^{n}\left\|\frac{\partial G}{\partial y_{i}}\right\|}}, i=1, \ldots, n
$$

the sign \pm depends on the sign of the derivative, i.e.,

$$
\begin{aligned}
& \frac{\partial G}{\partial y_{i}}>0 \leftrightarrow S_{f_{i}}>1, \\
& \frac{\partial G}{\partial y_{i}}<0 \leftrightarrow S_{f_{i}}<1,
\end{aligned}
$$

\section{Numerical results}

In the following example, we present a study on the reliability design optimization of the stator and the rotor of an ultrasonic motor with progressive wave SHINSEI USR 60 (figure 3), which is subjected to the constraint stress [15].

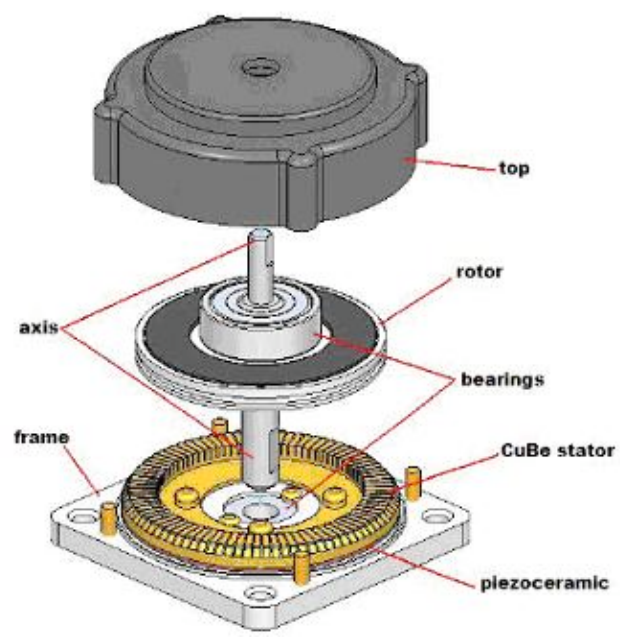

Fig. 3. Motor USR 60

\subsection{Geometry and operation}

The operation principle is described in figure 3. This motor is made of two main parts:

- The stator is a beryllium-coppper annular plate. At his circumference, teeth are machined to amplify the vibration movement and eliminate the wear particles. At his bottom surface; piezoelectric ceramics are glued to excite the metallic part. The stator is fixed to the frame at its center. To guarantee the free vibration of the stator ring, a decoupling fold is machined between the center and the circumference.

- The rotor can be separated in 3 zones: the axis, output of the motor; the track friction in contact with 
the stator and the spring fold linking axis to track and apply the rotor to the stator.

Other parts are the frame, the top and bearings. The piezoceramic ring is metalized on one face. The cube stator constitutes the ground electrode. This frequency is generally between 30 and $100 \mathrm{Khz}$, which justifies the name of ultrasonic motor (USM). Excitation at a natural frequency creates a traveling wave; each point of the top surface of the stator has an elliptic motion. The stator is in permanence in contact with the rotor. All these elementary displacements drive the rotor by friction. To have good contact conditions, a thin polymer layer can be reported on the rotor or the stator.

An approximation by finite elements is used. Figure $4 \mathrm{a}$ shows the model meshes of the stator and the rotor. It is realized with 900 hexahedral elements with 8 nodes per elements and 3 degrees of freedom per node. But the geometry of revolution of the structure and the axial loading of pre-stressing allows a two-dimensional axisymmetric model mesh (figure $4 \mathrm{~b}$ ). The grid of the structure is carried out with the help of the plane axisymmetric elements with 3 nodes and two degrees of freedom per node. The reduction of the structure's study to a two-dimensional axisymmetric problem allows a refined grid of these two surfaces while preserving the size of the system of equations after reasonable finite elements approximation. The body force $F_{\text {ext }}$ on the SHINSEI USR 60 motor, is transmitted to the rotor circumferential and punctually done at the ratio $\mathrm{R}=21.10^{-}$ ${ }^{3} \mathrm{~m}$ with the value $140 \mathrm{~N}$.

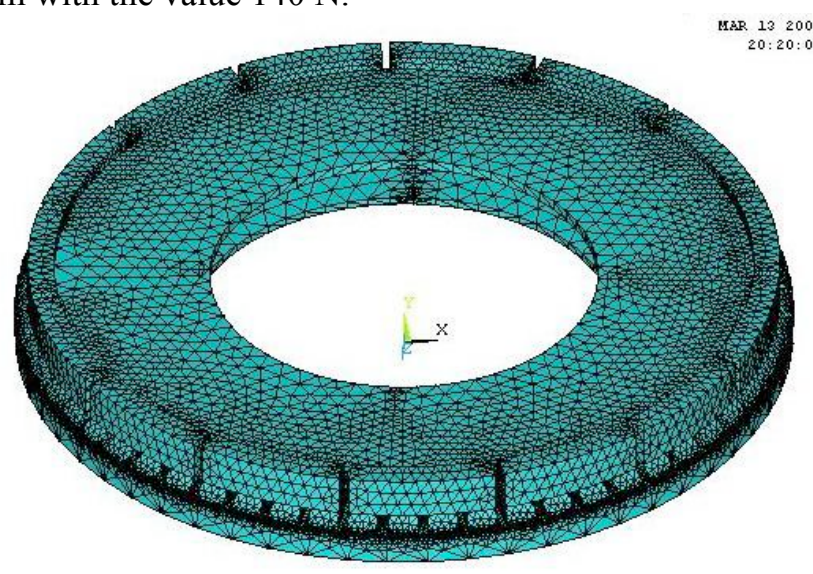

(a)Design domain

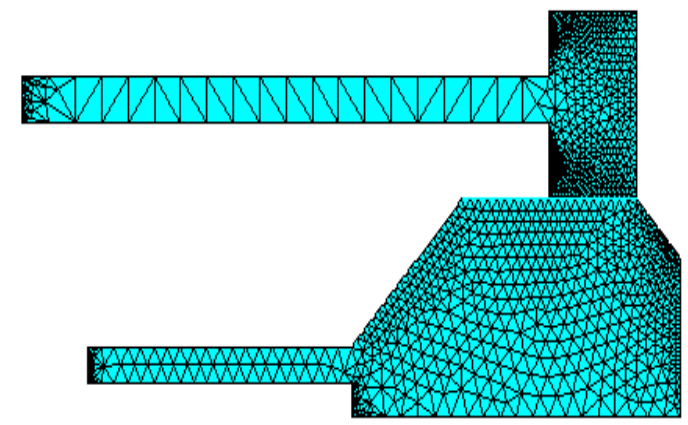

(b) Illustrative design
Fig. 4. Finite elements modelization of stator and rotor

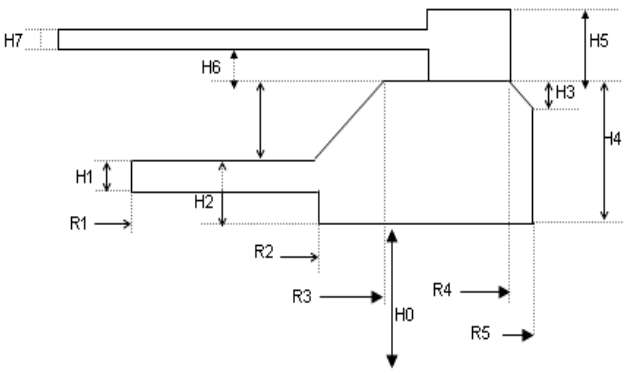

Fig. 5. Dimensional parameters of the stator and the rotor

The different dimensions of the stator and rotor are given in the table 1:

Table 1. Geometry characteristics

\begin{tabular}{cc}
\hline Parameters & Values $(\mathrm{mm})$ \\
\hline H0 & 0.5 \\
H1 & 0.6 \\
H2 & 1.2 \\
H3 & 1 \\
H4 & 4.2 \\
H5 & 3.2 \\
H6 & 1.3 \\
H7 & 2.1 \\
R1 & 16.5 \\
R2 & 22.5 \\
R3 & 25 \\
R4 & 29 \\
R5 & 30 \\
\hline
\end{tabular}

The mechanical characteristics of different materials are given in the table 2 :

Table 2. Materials data

\begin{tabular}{ccc}
\hline & Rotor & Stator \\
\hline $\mathrm{E}(\mathrm{MPa})$ & 72000 & 123000 \\
$\rho\left(\mathrm{Kg} / \mathrm{m}^{3}\right)$ & 2700 & 8250 \\
Poisson ratio & 0.3 & 0.31 \\
\hline
\end{tabular}

\subsection{Results and discussion}

The classical RBDO problem is written as follows: the optimization problem is to find the optimum value of the structural volume subject to the maximum stress (transient response). This problem can be expressed as:

$$
\begin{array}{cc}
\min & \operatorname{Area}(H 0, H 2, H 3, H 5, H 6, H 7) \\
\text { subject } & \sigma(H 0, H 2, H 3, H 5, H 6, H 7)-\sigma_{a d}=0 \\
\beta(x, y) \geq \beta_{t}
\end{array}
$$


where $\beta(x, y)=\min \left(\operatorname{dis}(u)=\sqrt{\sum_{i=1}^{6} u_{i}^{2}}\right)$.

The classical RBDO approach leads to a weak stability of convergence but the dynamic hybrid method allows the coupling between the reliability analysis and the optimization problem. The hybrid method problem can be expressed as:

$$
\begin{array}{cc}
\min & \operatorname{Area}(H 0, H 2, H 3, H 5, H 6, H 7) \cdot d_{\beta}(x, y) \\
\text { subject } & \sigma(H 0, H 2, H 3, H 5, H 6, H 7)-\sigma_{a d}=0 \\
& d_{\beta}(x, y, t) \geq \beta_{t}, \beta_{t}=3.8
\end{array}
$$

where $\mathrm{H} 0, \mathrm{H} 2, \mathrm{H} 3, \mathrm{H} 5, \mathrm{H} 6$ and $\mathrm{H} 7$ are grouped in the random vector y but to optimize the design, the means $\mathrm{m}_{\mathrm{H} 0}, \mathrm{~m}_{\mathrm{H} 2}, \mathrm{~m}_{\mathrm{H} 3}, \mathrm{~m}_{\mathrm{H} 5}, \mathrm{~m}_{\mathrm{H} 6}$ and $\mathrm{m}_{\mathrm{H} 7}$ are grouped in the deterministic vector $\mathrm{x}$, and their standard-deviation equals to $0.1 \mathrm{~m}_{\mathrm{x}}$.

In our case, OSF method proceeds in the following steps:

i) Determine the design point (the most probably point of failure). We minimize the right section of the rotor-stator under the design constraint without the safety factors. The optimization problem can be written as follows:

$$
\begin{array}{cc}
\min & \operatorname{Area}(H 0, H 2, H 3, H 5, H 6, H 7, R 2, R 3, R 4) \\
\text { under } & \sigma_{\text {eqv }}(H 0, H 2, H 3, H 5, H 7, R 2, R 3, R 4)-\sigma_{c}=0
\end{array}
$$

The von Mises equivalent constraint of the computed optimal solution is equal to $235.24 \mathrm{MPa}$ which is $0.21 \%$ greater than the elasticity limit.

ii) Compute the optimal safety factors by using th formula 13 .

In this application, the number of the deterministic variables is equal to the random variables. The sensibility values of the limit state corresponding to the optimization variables are obtained during the optimization process. The different results of the optimal safety factors relative to each design optimization variables are given in the table 3:

Table 3. Results of the optimal safety factors

\begin{tabular}{llll}
\hline Variables & $\frac{\partial G}{\partial y_{i}}$ & $u_{i}$ & $S_{f_{i}}$ \\
\hline H0 & 1.01 & 0.217 & 1.021 \\
H2 & -0.254 & 0.109 & 0.989 \\
H3 & -0.136 & 0.080 & 0.992 \\
H5 & -1.178 & 0.234 & 0.976 \\
H6 & 11.470 & 0.730 & 1.073 \\
H7 & -292.4 & 3.695 & 0.630 \\
R2 & -0.067 & 0.055 & 0.994 \\
R3 & -0.216 & 0.1005 & 0.989 \\
R4 & 2.463 & 0.339 & 1.034 \\
\hline
\end{tabular}

iii) Evaluate the optimal solution by introducing the values of the safety factors in the values of the design variables.

RBDO results using the optimal safety factors are given in the tables 4 and 5:

Table 4. Results of the OSF method

\begin{tabular}{lcl}
\hline Variable & Design point & $\begin{array}{l}\text { Optimal } \\
\text { solution }\end{array}$ \\
\hline H0 & $0.52195 \times 10^{-3}$ & $0.53998 \times 10^{-3}$ \\
H2 & $0.11563 \times 10^{-2}$ & $0.12749 \times 10^{-2}$ \\
H3 & $0.66719 \times 10^{-3}$ & $0.99276 \times 10^{-3}$ \\
H5 & $0.32564 \times 10^{-2}$ & $0.24628 \times 10^{-2}$ \\
H6 & $0.12530 \times 10^{-2}$ & $0.13973 \times 10^{-2}$ \\
H7 & $0.20121 \times 10^{-2}$ & $0.20821 \times 10^{-2}$ \\
Constraint & $0.23564 \times 10^{9}$ & $0.23530 \times 10^{-2}$ \\
Surface & ------ & $0.41405 \times 10^{-4}$ \\
Reliability & 3.6 & ----- \\
index & & \\
\hline
\end{tabular}

Table 5. Results of the OSF method

\begin{tabular}{lll}
\hline Variable & $\begin{array}{l}\text { Design } \\
\text { point }\end{array}$ & $\begin{array}{l}\text { Optimal } \\
\text { solution }\end{array}$ \\
\hline H0 & $0.53998 \times 10^{-3}$ & $0.5513 \times 10^{-3}$ \\
H2 & $0.12749 \times 10^{-2}$ & $0.126 \times 10^{-2}$ \\
H3 & $0.99276 \times 10^{-3}$ & $0.983 \times 10^{-3}$ \\
H5 & $0.24628 \times 10^{-2}$ & $0.230 \times 10^{-2}$ \\
H6 & $0.13973 \times 10^{-2}$ & $0.149 \times 10^{-2}$ \\
H7 & $0.2082 \times 10^{-2}$ & $0.1311 \times 10^{-2}$ \\
Constraint & $0.2358 \times 10^{9}$ & $0.1560 \times 10^{9}$ \\
Surface & ------- & $0.5635 \times 10^{-4}$ \\
Reliability & 3.8 & ----- \\
index & & \\
\hline
\end{tabular}

To search the solution using the deterministic design optimization one, we have considered one safety factor. The founding solution does not respect the desired safety level, so we must use the OSF for better numerical/computing result. With this method, we obtain one optimal solution with a good reliability index (3.8).

\section{Conclusions}

A RBDO solution that reduces the structural weight in uncritical regions both provides an improved design and a higher level of confidence in the design. The classical RBDO approach can be carried out in two separate spaces: the physical space and the normalized space. Since very many repeated searches are needed in the above two spaces, the computational time for such an optimization is a big problem. The structural engineers do not consider the RBDO as a practical tool for design optimization. Fortunately, an efficient method called the Hybrid Method (HM) has been elaborated where the optimization process is carried out in a Hybrid Design Space (HDS). However, the vector of variables here contains both deterministic and random variables. The RBDO problem by HM is thus more complex than that 
of deterministic design. The major difficulty lies in the evaluation of the structural reliability, which is carried out by a special optimization procedure. The procedure of HM is still complex to be implemented on the machine that can provide a big part of the RBDO problem analytically (high reduction of the computing time). Based on the reduction of the computing time, we proposed here a new methodology called safety factors method. The OSF methodology is based on reliability and sensitivity analysis, and contains the advantages of both the reliability-based and the deterministic design procedures. The advantage of the former is the satisfaction of a required reliability level (target reliability index) and the advantage of the latter is that it is no need for additional computational time because the designer can use the results of the sensitivity analysis of the constraint function obtained during the optimization process when we consider the deterministic variables as random ones.

Please edit the final version of your paper strictly complying with the template.

An integrated layout optimization method is proposed in this paper to deal with the simultaneous design of structure and support layout. Based on the previously proposed layout design techniques of multi-component system, the supports are considered as solid components that are partially embedded into the design domain.

\section{References}

1. O.A. Baba, B. Radi, J.-C. Gelin, An Augmented Lagrangian Treatment of the metal forming process. Math. and Comp. Model. 32(10), 171-179, (2000).

2. T. Moro, A. El Hami, A. El Moudni, Reliability analysis of a mechanical contact between deformable solids. Prob. Eng. Mech. 17(3), 227-232, (2002).

3. A. El Hami, T. Moro, Une analyse par la fiabilité des structures des problèmes d'usure du couple stator/rotor dans un moteur piézo-électrique onde progressive. Sûr. de Fonct. 24(2), 21-34, (2003).

4. HJ. E. Rojas, A. El Hami, D. A. Rade, Reliability analysis based on gradient and heuristic optimization techniques of composite laminates using element-free Galerkin method. Int. J. for Sim. And Mult. Design Opt. 2(2), 157-169, (2008).

5. N. Kikuchi and J.T. Oden, Contact problems in elasticity: a study of variational inequalities and finite element methods, (SIAM: studies in applied mathematics, 1988).

6. Y. S. Feng, F. Moses, A method of structural optimization based on structural system reliability. J. Struct. Mech. 14, 437-453, (1986).
7. J. Tu, K. K. Choi, Y. H. Park, A new study on reliabilitybased design optimization. J. of Mech. Design 121(4), 557-564, (1999).

8. B. D. Youn, K. K. Choi , Y. H. Park, Hybrid analysis method for reliability-based design optimization. J. of Mech. Design 125(2), 221-232, (2003).

9. B. D. Youn, K. K. Choi, L. Du, Adaptive probability analysis using an enhanced hybrid mean value method. Struct. Multidisc. Optim. 29, 134-148, (2005).

10. O. Bendaou, A. El Hami , A. Aannaque, M. Agouzoul, Calculation time optimization for stochastic analysis of an industrial structure. Int. J. for Sim. and Multidisc. Design Opt. 2(2), 135-141, (2008) .

11. G. Kharmanda, A. Mohamed, M. Lemaire, Efficient reliability-based design optimization using hybrid space with application to finite element analysis. Struct. and Multidisc. Opt. 24, 233-245, (2002).

12. G. Kharmanda, A. El Hami, N. Olhoff, Global Reliability-Based Design Optimization, In Frontiers on Global Optimization, C. A. Floudas, ed. (Kluwer Academic Publishers) 255, (2004)

13. G. Kharmanda, N. Olhoff, A. El Hami, Optimum safety factor approach for reliability- based design optimization with extension to multiple limit state case. Struct. and Multidisc. Opt. 26, 45-60, (2004).

14. G. Kharmanda, A. Mohsine, A. Makloufi, A. El Hami, Recent methodologies for reliability-based design optimization. Int. J. for Sim. and Mult. Design Opt. 2(1), 1124, (2008).

15. P. Le Moal, P. Cusin, Optimization of a traveling wave ultrasonic motors using a three dimensional analysis of the contact mechanism at the stator-rotor interface. Eur. J. of Mech. A, 18, 1061-1084, (1999). 